\title{
NETBALTIC SYSTEM - HETEROGENEOUS WIRELESS NETWORK FOR MARITIME COMMUNICATIONS
}

\author{
Michał Hoeft \\ Krzysztof Gierłowski \\ Jacek Rak \\ Józef Woźniak \\ Gdańsk University of Technology, Poland
}

\begin{abstract}
In case of maritime communications, we observe a growing interest in deployment of multitask satellite-based solutions and development of new maritime-specific systems intended for improvements in safety of e-navigation. Analysis of different types of currently used maritime communication systems leads, however, to a conclusion that neither global and still very expensive satellite systems nor cheaper, but short-ranged transmission technologies can, on their own, fully meet the today's expectations and quality requirements formulated for broadband maritime systems. This lack of reliable solutions, offering high throughput and ubiquitous availability of coverage to a wide audience at a relatively low price is one of the main barriers in a widespread implementation of e-navigation initiatives.

This issue is addressed in the netBaltic project with the objective to design, deploy and validate in a real maritime environment a non-satellite wireless communication system enabling ship-to-ship and ship-to-shore information exchange via a multi-hop network composed of onshore base stations, maritime vessels and other transit elements such as buoys.

In this paper, the idea of a heterogeneous wireless maritime system is presented. Details of the proposed netBaltic node architecture are described highlighting the solutions introduced in the project as a response to specific maritime communication requirements. Numerical results of communication area coverage are presented for four different scenarios utilizing different wireless transmission technologies. In particular, they indicate that when using appropriate wireless communication solutions, the number of vessels being able to connect to Internet is significantly increased as compared to traditional wireless systems (capable of one-hop communication) from $14 \%$ for short-range transmission technologies up to as high as $127 \%$ in case when relatively long-range transmission technologies are employed within the system.
\end{abstract}

Keywords: maritime communication systems,heterogeneous maritime network,mobility management,maritime mesh networks,delaytolerant networks

\section{INTRODUCTION}

Maritime communication systems are expected to support e-navigation services, enable voice/video transmission, as well as information exchange (e.g., email or web browsing) via the global information infrastructure (Internet [1,2]). As pointed out in [3], about 80 percent of the worlds' trade is transported by maritime units, making the importance of maritime communications evident. Additionally, a number of other marine activities including e.g., fishing, sea exploitation or tourism increase the significance of marine communication systems and the necessity of broadband communications even more.
Analysis of IMO (International Maritime Organization) mandated systems and services shows that the current approach has been to provide a set of critical safety-related services, that have been designed and standardized to be reliably maintained using very low throughput data channels [4]. Supplementary services, such as download of digital navigation maps, frequent update of weather maps [5], automatic route optimization or various remote monitoring and maintenance services, not to mention Internet access for improving ship crew and passenger comfort, requires significantly more efficient communication capabilities and has not been as strictly standardized due to lack of comprehensive, reliable solutions. 
Several years ago, IMO proposed a concept known as e-navigation $[1,6]$. This concept harmonizes the collection, integration, exchange, presentation and analysis of maritime information onboard and ashore by electronic means to enhance berth-to-berth navigation and related services, for safety and security at sea and protection of the marine environment. A high-speed and cost-effective maritime wireless communications are essential for the success of the above concept as the deployment of a wide range of e-navigation solutions requires broadband or semibroadband transmission capability.

In addition to bandwidth requirements of e-navigation services, high demand for capacity also comes from ship crews which are demanding Internet access to stay connected with family and friends. Provisioning of highly demanding realtime applications within a distinct operational environment (which in our case is a continuously changing wireless network over sea area) is expected to bring up significant challenges.

Currently, the majority of solutions which can be expected to provide necessary long range ship-to-ship and ship-to-shore communications, require relatively expensive satellite solutions, so their usability is effectively limited to large maritime units: ferries and some number of SOLAS (International Convention for the Safety of Life at Sea) class ships. Satellite communication, apart from its high cost, is also characterized by a high communication latency (over $500 \mathrm{~ms}$ - in cases of popular geostationary satellite systems) and a low transmission rate. Therefore, it is not appropriate for a number of applications [7].

Among currently deployed services that can be provided without satellite communications involved, one can mention the identification and location of vessels assured by the low-rate Automatic Identification System (AIS), as well as the Global Maritime Distress and Safety System (GMDSS) [8-10]. A clear disadvantage of the existing non-satellite communication systems based on High Frequency (HF), Very High Frequency (VHF) and Ultra High Frequency (UHF) radios is that they provide remarkably slower transmission than popular, shore-based wireless networks [4]. Wide analysis of maritime communication systems and scenarios is presented in [11].

The existing HF/VHF systems will be significantly extended and/or partly replaced by a new VHF Data Exchange System (VDES) currently being developed by IMO, IALA (International Association of Marine Aids to Navigation and Lighthouse Authorities) and ITU (International Telecommunication Union). The work leading to a specification of the VDES has been initiated due to the need to address a growing overload of VHF Data Link (VDL) of the AIS and an intent to enable a wider and seamless data exchange for the maritime community [12-14]. With possible data rates of about $200 \mathrm{~kb} / \mathrm{s}$, the system can be expected to facilitate numerous applications for safety and security of navigation, protection of marine environment, efficiency of shipping etc. However, it still cannot be called a broadband solution capable of supporting modern network services.
Lack of such a communication system has stimulated a number of research and implementation projects to be undertaken, seeking to employ inexpensive, broadband communication solutions, such as popular Wireless Local Area Network (WLAN) and Wireless Metropolitan Area Network (WMAN) technologies in maritime environment. For these technologies to be successfully employed in maritime environment, their deployment is most often limited to a specific set of scenarios, where they are able to provide users with a clearly defined, predictable and reliable service level. Initiatives such as WISEPORT (WIreless broadbandaccess for SEaPORT) network in Singapore as well as TRImedia Telematic Oceanographic Network (TRITON) project [15] are good examples of such an approach, able to provide a high-speed and low-cost maritime communications in maritime areas close to the shore. A review of satellite and non-satellite maritime oriented systems has been presented in [16].

All these systems, however, concentrate on deployment of a single specific communication technology for the terrestrial ship-to-ship and ship-to-shore communications, thus creating a homogeneous systems utilizing a modified (non-standard) version of employed technology. In contrast, the main aim of the netBaltic project is to develop and deploy a broadband wireless communication system providing connectivity in a heterogeneous wireless environment (consisting of a combination of multiple, standard wireless communication devices), able to meet the requirements of e-navigation services.

The paper is organized as follows. In the next section, related works are discussed presenting the comparable proposals of maritime communication systems. After that, details of the netBaltic system are introduced with presentation of the netBaltic system and node architecture. Analysis of network topologies for different scenarios and discussion of results are presented in the subsequent section, followed by conclusions.

\section{RELATED WORKS}

A wide range of applications (such as Internet access, data/voice transmission or e-navigation) requires highspeed communications every time and everywhere they are used, thereby creating the need for low-cost non-satellite communication solutions as an alternative to existing satellite maritime systems.

A set of available proposals of wireless non-satellite communication techniques is rather limited, and they mostly remain in the conceptual stage described in research papers. Their major focus is put on enabling the non-satellite maritime communications of a ship-to-shore type at increased transfer rates and communication coverage. However, they are still constrained by their significantly limited communication range from the on-shore infrastructure [17], meaning that they can be beneficial only in specific areas such as ports.

A limited set of proposals that have been validated in real scenarios includes e.g., the MariComm system [17] using the 
high-gain directional antennas to extend the transmission range and to be able to obtain the full $360^{\circ}$ coverage in azimuth. In MariComm, the transfer rate is provided at the level of about $1 \mathrm{Mbps}$, within max. $100 \mathrm{~km}$ from the shore. More distant areas are not covered by this system.

Researchers commonly propose to utilize the IEEE 802.16 [18] specification (referred to as WiMAX) to increase the transmission rate up to several tens of Mbps between devices separated by distances of up to $40 \mathrm{~km}$ (or more, if extensions of the base standard such as IEEE 802.16m [19] are applied). Following [20], most of maritime applications could be efficiently served by utilizing a $10 \mathrm{Mbps}$ rate communication system. The example of such an approach, a maritime communication architecture based on the Wireless Mesh Network (WMN) communication paradigm presented in [7], uses long-range WiMAX networking solutions to set up a WMN providing IP-based, real-time ship-to-shore and shipto-ship communications. The proposal described in [7] also aims to reduce the negative influence of mobility of vessels and resulting frequent changes of a network topology by using AIS to access information related to movement patterns of network nodes to simplify the process of planning and updating of a dynamic network topology.

The TRITON project $[15,20]$ resulted in the development of a high-speed wireless mesh network formed between ships, marine beacons and buoys enabling the ship-to-shore and ship-to-ship communications based on IEEE 802.16 [18] standards. To overcome the issues of sea surface movement and sea wave reflections, advanced MIMO solutions have been deployed allowing for $360^{\circ}$ reception and transmission.

Another proposal, being an outcome of an international collaboration between Institute for Infocomm Research, Agency for Science, Technology \& Research, Singapore and National ICT Australia [21], is a merge of the high speed maritime mesh technology (based on TRITON) with legacy satellite communications by means of an abstraction layer called "intelligent middleware", which also performs link specific protocol optimizations. The system automatically switches to satellite communications when ships are too sparse or too far from the onshore switching units.

It can be seen that developers of the abovementioned solutions have chosen a single wireless transmission technology and concentrated on adapting it for the most efficient use in maritime conditions, often introducing nonstandard modifications of its core mechanisms (for example: to enable multi-hop transmission capability) or obligatory hardware elements, as e.g., dedicated antenna sets. Such an approach enables the use of mechanisms dedicated to address specific characteristics of a particular technology (which often allows for a higher efficiency of the resulting solution) and the homogeneity of the system makes its operation more predictable. However, it is necessary for all users of the system to use a specific hardware solution, including a nonstandard wireless transmission system (possibly the most expensive part of the system's shipboard installation). The costs involved can be a limiting factor in popularization and wider deployment of a given system, which in case of WMN solutions (requiring a specific minimum density of nodes to form a consistent network structure) is a serious drawback.

\section{NETBALTIC SYSTEM ARCHITECTURE}

The netBaltic project aims to design, deploy and validate in a real maritime environment, a non-satellite wireless heterogeneous communication system enabling ship-toship and ship-to-shore information exchange via a multihop network composed of onshore base stations, vessels and other transit elements such as buoys. In contrast with the abovementioned initiatives, the netBaltic system has been designed to provide a comprehensive, maritime communication solution available to users regardless of their current location in relation to an on-shore network infrastructure or other maritime vessels. It is evident that no currently available broadband transmission technology is capable of providing such ability by itself, including popular: - Wireless Local Area Networks (WLANs) such as a multitude of WiFi technologies providing different transmission ranges and rates (IEEE $802.11 \mathrm{a} / \mathrm{b} / \mathrm{g} / \mathrm{n} / \mathrm{ac}$ $[22,23])$,

- Wireless Metropolitan Area Networks (WMANs), including different variants of WiMAX technology (IEEE $802.16 \mathrm{~d} / \mathrm{e}[24,25]$ ) and various proprietary solutions (for example: AirMAX [26], RADWIN Fiber-In-Motion [27]),

- Wireless Wide Area Networks, represented by 3GPP standardized technologies, such as $3 \mathrm{G}$ or LTE cellular solutions.

In this case, the netBaltic project employs an innovative multi-zone approach (Fig. 1), which allows it to utilize different types of network organization principles and specific mechanisms depending on communication capabilities currently available in a specific maritime area: a mobilityaware point-to-multipoint access network, a self-organizing mesh network and a delay-tolerant network (DTN). Such a solution allows the system to differentiate its operation procedures to utilize the available communication resources in the most efficient manner possible.

To facilitate a deployment of the netBaltic system, a complete set of its core mechanisms has been implemented in the ISO-OSI network layer, which allows any communication technology capable of transmitting IP datagrams to be transparently utilized. As this requirement is fulfilled by a majority of currently available, off-the-shelf communication technologies including a complete set of diverse network solutions mentioned above, the netBaltic system allows for creation of a vastly heterogeneous communication system. Even low-bandwidth HF/VHF maritime data transmission systems can be used if more efficient alternatives are not available. Moreover, the ability to transparently utilize different transmission technologies permits the system to naturally evolve, improving its coverage and data transmission capabilities as new technologies become available.

Each of the three abovementioned network organization principles is utilized within its specific zone with its area 
of coverage dynamically changing depending on current propagation conditions, spatial placement of system nodes and their specific communication capabilities (i.e. supported transmission technologies).

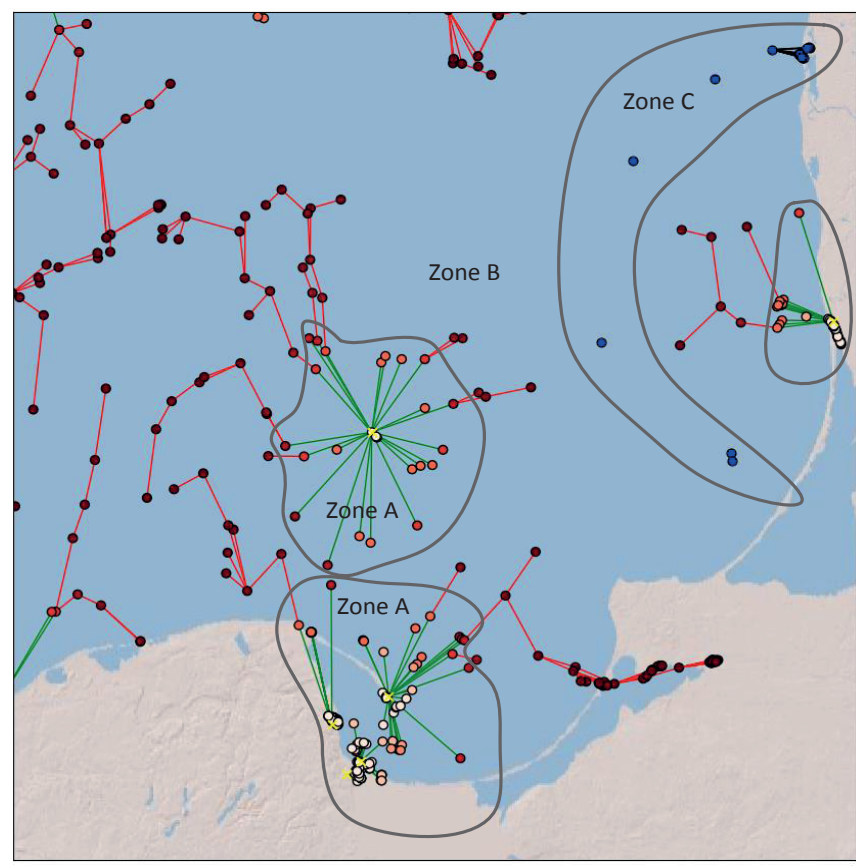

Fig. 1. A multi-zone structure of the netBaltic system. On-shore dependent zone $A$ is also present in vicinity of Petrobaltic oil rig

When the network node is located within range of the on-shore infrastructure with its reliable and efficient access networks maintained by various network providers (zone A), it will operate as a universal access client. All of its communication interfaces will be used to detect the available access networks and their base stations (or access points). The node (a central unit with wireless interfaces) will then connect to one or more of such networks following a policy set by its user and taking into account their communication capabilities and quality provided. The great number of interfaces can be used and there is no limitation in the proposed architecture.

However, as the location of the node changes or due to changing traffic conditions within chosen access networks, the initial network selection will become outdated and quality of communications will degrade making it necessary to repeat the selection process. The resulting change of the access network utilized by the terminal (handover) would normally cause a significant degradation of transmission parameters, as the mobile node terminates its current connection and then reconnects to a new network, introducing an additional time interval with no connectivity. Moreover, the new network may require a change of the node's IP address, effectively terminating any ongoing application sessions, such as data transfers, video streaming or audio calls. These adverse effects of a handover process result in standard communication terminals performing it only when strictly necessary (for example, when the existing connection has been lost), thereby foregoing the ability to employ available communication resources in an efficient manner.
To address these limitations, the netBaltic system employs a soft-handover and network-layer mobility management procedures within its zone A [28], allowing both uninterrupted application sessions and highest available Quality of Service to be provided. By utilizing multiple communication interfaces, the node is able to obtain information about alternate access networks prior to the point when handover becomes necessary due to a loss of the current link. Moreover, it is possible to establish a new communication link before disconnecting the currently used one, which practically eliminates the connectivity-loss interval.

Despite the above-described solution, it is most often the case that the network address and other network stack configuration parameters are subject to change as a result of a handover process. To prevent user network sessions from being disrupted by such a change, the netBaltic node zone A mechanisms incorporate a network layer mobility management solution, allowing the node to retain the same network address despite moving between different access systems $[29,30]$ while the incoming traffic is always forwarded to its current location.

The described set of mechanisms allows the netBaltic node to freely move within zone A seamlessly changing its point of network access without any disruption of user network sessions [29]. This ability, due to lack of handoverrelated interruptions, allows the node to aggressively change its network access technology and provider to maximize the quality of network service level available for the user or to follow other user-specified policies - for example the minimization of costs.

If the netBaltic network node leaves the coverage area of infrastructure-dependent access systems, it can still retain a real-time network access by employing a self-organizing mesh network mechanisms used in netBaltic zone B. In this zone, all netBaltic nodes perform detection of other nodes within their communication range and establish inter-node links when possible, thus dynamically forming a network structure. Within such a structure any node will forward data packets intended for remote destinations, allowing all participating nodes to communicate (Fig. 2). Moreover, if any of such nodes has a connection to the external network (for example if it also belongs to zone $\mathrm{A}$ and has a direct connection to Internet with use of the on-shore base station), it is capable of serving as a data exchange point for all other nodes within the mesh structure. If present, such a data exchange point will provide nodes within zone B with external connectivity (for example Internet access) and capability to integrate separate partitions of zone B network structure (Fig. 2). The system in zone B has been designed to maximize its coverage area and attainable network throughput, thus allowing a maximum possible number of maritime vessels to obtain a broadband communication capability. The decision to maximize coverage and throughput at the cost of other QoS parameters (such as transmission delay and jitter) has been made as a result of requirements analysis of both currently deployed and planned e-navigation services, as well as those of a number of Internet services popular in maritime community. 
Due to a mobile nature of maritime vessels and changing propagation conditions, the structure of a network within zone B can be expected to undergo relatively frequent changes as inter-node links are established and lost. Efficient operation of a multi-hop mesh network in such an environment requires a significant number of specialized mechanisms capable of creating and maintaining both the described network structure and dynamically selected multi-hop data transmission paths. The most crucial ones include: mesh neighborhood discovery, intra-node link management and quality assessment, data transmission path discovery with procedures for recovery from node/link failures and multihop data forwarding [29].

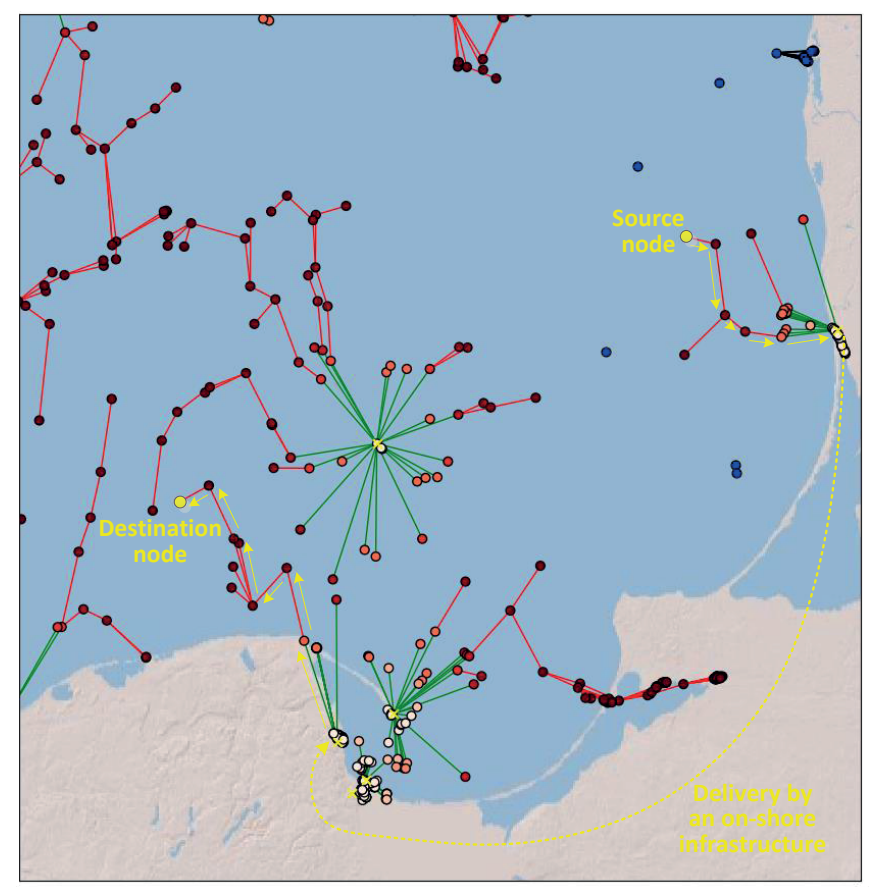

Fig. 2. Multi-hop data delivery and integration of zone B partitions

Such a self-organizing multi-hop network structure allows groups of ships to communicate over much longer distances than any single broadband transmission technology could provide, as long as their spatial placement permits the required network structure to be formed. It is expected (as shown later in this paper) that with the transparent support of a wide range of popular transmission technologies, zone B network structure would be able to extend the system's connectivity coverage outside zone A over frequently used areas such as harbor approaches and shipping lanes. The ability to integrate a wide range of communication technologies already available on a given vessel, combined with the ability to transparently employ both inexpensive off-the-shelf and highly-capable, specialized communication solutions, makes the system a viable solution for a very wide group of users. Such a variety of deployment options and user-based far surpassing systems requiring the use of (costly) non-standard WMAN technologies, is expected to allow the system to reach significantly higher node densities - a crucial ability for a self-organizing wireless mesh network depending on client devices to form a consistent network structure.

Of course, in the case of areas only sparsely occupied by vessels, density of system nodes will likely be insufficient to form a structure of zone B mesh network, and if they are located far away from the shore infrastructure, no real-time network communications will be possible. To address the communication requirements of users located in such areas (for example of data-recording buoys), the netBaltic system defines a set of Delay Tolerant Network (DTN) mechanisms, allowing its nodes to prepare data packages of a significant size (tens of MBs), which will be stored by the system nodes and exchanged between vessels (as they pass each other within the communication range), to eventually reach their intended recipient.

This functionality has been implemented in an overlay manner, which means that it is available in all zones. Also, it is subject to a decision of an application whether to use the DTN functionality or not. In this situation, zone C is defined as an area where no communication method other than DTN is supported by the system.

The described functionality of a multi-zone netBaltic system requires a significant number and complexity of employed mechanisms and a careful management of their interactions to ensure a seamless integration. The general architecture of the netBaltic node networking stack is presented in Fig. 3.

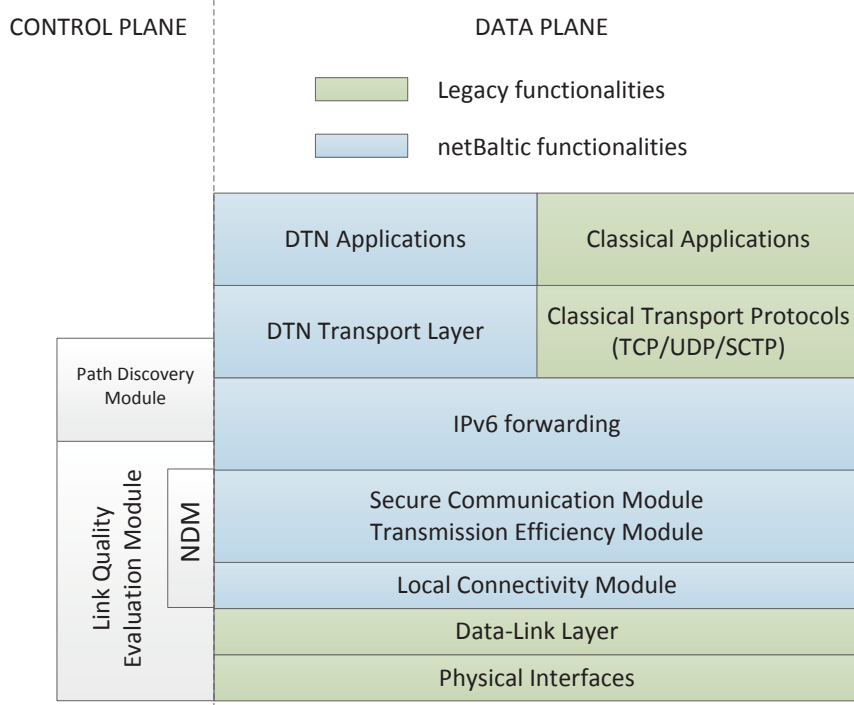

Fig. 3. Networking stack of the netBaltic node

Functional relations between netBaltic node modules are presented in Fig. 4. Green lines refer to connections in the control plane, whereas blue lines show connections in the data plane. 


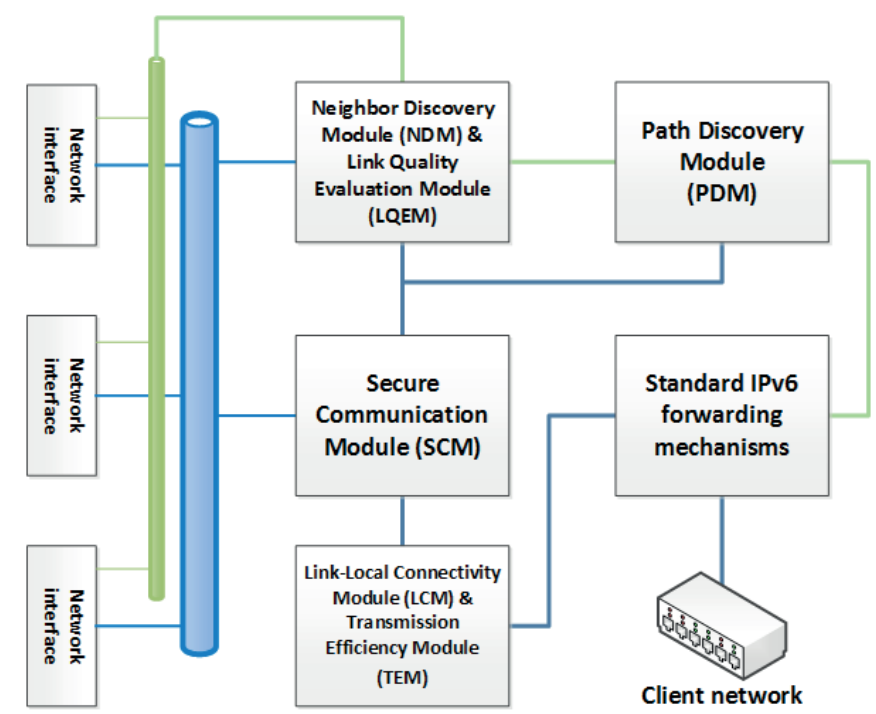

Fig. 4. Functional relations between netBaltic node modules

It can be seen that the above architecture incorporates a number of legacy functions present in Linux operating system specifically in its data plane part. The authors aim was to utilize the already developed and well-tested solutions when possible (such as a standard IPv6 packet forwarding mechanism) supplementing them with additional functions and a control logic necessary for the operation of the system.

The control plane contains elements (see Fig. 3 and Fig. 4) that are specific to netBaltic exclusively, of which the most universally employed one is a Link Quality Evaluation Module (LQEM). This module is responsible for gathering quality-related information regarding each access (zone A) or inter-node link (zone B) which a given node is capable of establishing. This information is used to calculate a metric referred to as Link Quality Indicator (LQI) allowing the node to estimate the data-transmission rate of a given (already established or only considered) link. While the most precise estimation of LQI value requires gathering transmission technology-dependent information, a node is also capable of an estimation of the LQI value using only internal measurement techniques implemented in the network layer. Link Quality Indicator is used in a decision making phase of the mobility management solution implemented in the netBaltic system (zone A) and in the process of both mesh link establishment and data transmission path selection (zone B).

A Neighbor Discovery Module (NDM) is utilized by mesh network mechanisms and allows the node to detect the other neighboring nodes of the system and advertise its own presence. The LQI of possible inter-node links is subsequently evaluated, which can result in a decision to add them to zone B network structure.

With the mesh structure of inter-node connections provided by the NDM, a dedicated Path Discovery Module (PDM) is responsible for utilizing it for establishment of multi-hop data transmission paths. For this purpose, it employs a hybrid solution, which combines a proactive and a reactive path discovery $[29,30]$. As the expected traffic patterns include a major portion of the traffic being exchanged with external networks (especially the Internet), a proactive approach is used in this case - a tree-based routing solution, in which on-shore stations periodically broadcast their presence, causing transmission paths to be formed towards them. Such a solution allows the continuously updated set of data transmission paths connecting all system nodes with network gateways to be constantly maintained. In the case of communication between nodes within the mesh network a reactive procedure is used - specifically a modified version of the AODV protocol [31]. This approach prevents from an excessive amount of management traffic from being generated, as such paths are discovered only when they are specifically required. In all cases, LQI link metric (representing an assessment of link's expected throughput) is used in the path selection process. The LQI is calculated taking into account both current and historical performance of the link, to include a measure of its stability in the resulting value. The measurements useful for LQI calculation include a selection of wireless system's parameters (for example: received signal strength, signal-to-noise ratio, connectivity gaps), but it is possible to calculate it based exclusively on network-level (IP) traffic flows.

Another important group of netBaltic-specific elements can be seen near the bottom of the networking stack. These modules are responsible for a seamless integration of transmission technologies with the network layer functionality of the node, including additional procedures addressing the efficiency of data transmission over wireless links in a maritime environment. Link-Local Connectivity Module (LCM) and Transmission Efficiency Module (TEM) perform a complete set of functions necessary to conduct an efficient delivery of IPv6 packets which have been selected for transmission over a specific inter-node link. The LCM functions include adding/removing of necessary packet headers and performing header compression, while TEM ensures a reliable packet transfer through the link by employing an acknowledged transmission method and appropriate buffering. Such an approach has proven to be necessary for an efficient operation of a multi-hop network in a maritime environment due to the occurrence of periodic link degradation intervals caused by sea wave motions. Several solutions are currently being evaluated for this purpose, including ARQ mechanisms, network coding and tunneling over a streaming protocol (such as TCP or URP).

Communication security functions of our heterogeneous system including authentication, confidentiality and integrity, are provided at the inter-node link level by a Secure Communication Module (SCM). Their operation takes advantage of a mature IPsec standard [32] supplemented with dedicated management procedures providing reliable identity management (including X.509 certificates enrollment, distribution and revocation) in all three netBaltic networking environments.

The DTN procedures of netBaltic form a separate group of mechanisms possible to be employed by DTN-aware applications regardless of the vessel's location. They operate 
using an overlay model over the IPv6 network provided by mobility-aware access or mesh networks described in this paper.

For the purpose of delivery of DTN data packages to their destinations, a loosely synchronized database of node locations is commonly created based on information received by vessels using Automatic Identification System (AIS) [8]. A set of procedures allows the system to limit the geographical area over which a given package is distributed and the amount of time the package is retained in different node storage space. The procedures employed will allow its delivery even if the location data is highly outdated by sequentially extending the delivery area and eventually falling back to a global Epidemic DTN delivery [33].

\section{SYSTEM MODELLING AND EVALUATION RESULTS}

To evaluate the operational scope of the proposed system, numerical evaluations were conducted, aiming to assess a maximum throughput of ship-to-shore communication available to maritime vessels employing netBaltic communication terminals. The necessary input data has been obtained by means of real-world measurement campaigns conducted in a marine environment and by employing historical AIS (Automatic Identification System) information available from maritime authorities. AIS is a safety system used by vessels and buoys to broadcast information about their locations, destination ports and estimated time of arrival, as well as the ship's tonnage, its course and speed, in order to improve the safety of navigation. Communication hardware parameters of the devices employed are presented in Table I. Table I. Evaluation parameters

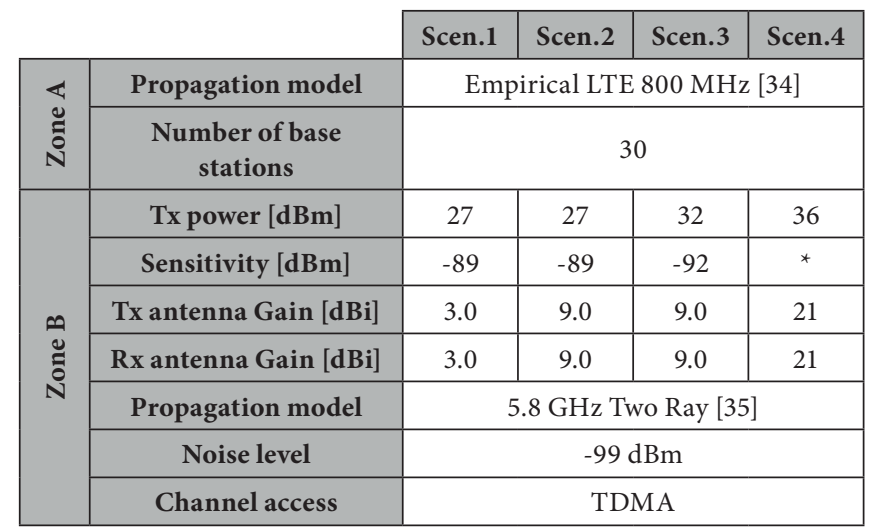

*) due to limited availability of technical information regarding a proprietary technology (Kongsberg Maritime Broadband Radio [36]), the maximum communication range has been assumed to be a manufacturer-provided value.

In evaluation, the authors have considered different wireless technologies for ship-to-ship and ship-to-shore communication links. As a solution used for direct communication in zone A (ship-to-shore), LTE connections were employed. The bandwidth over such connections has been calculated in accordance with the model obtained during the real measurement campaigns conducted as a part of the netBaltic project. As a result, a function describing the effective link bandwidth depending on a distance between a vessel and a base station has been calculated (see Fig. 5). For distance values from within the range covered in measurements, the isotonic regression has been used to find the relation between bandwidth and distance. For distance values outside this range, a linear regression has been employed. In case of zone B (ship-to-ship links), a proprietary wireless technology operating in $5.8 \mathrm{GHz}$ band has been employed - RADWIN Fiber-In-Motion [27]. The exception is Scenario 4, where in place of a relatively short range/high-bandwidth RADWIN WMAN, a long-range communication technology (Kongsberg Maritime Broadband Radio [36]) is used for links between vessels within zone $B$.

Each of these four scenarios uses different configuration of a transmitter (i.e. transmit power), a receiver (sensitivity) and antennas (gain), to illustrate different capabilities of radio communication set-ups available to netBaltic network nodes. All of these parameter sets are kept within capabilities of the equipment currently available off-the-shelf. The link-rate for each wireless link has been calculated by means of the adaptive modulation scheme with thresholds being the same as in a single chain IEEE 802.11ac [23], which is also appropriate for RADWIN proprietary technologies considered in the paper.

For each vessel, a best path to all reachable netBaltic on-shore base stations has been calculated and from this set a path with the best metric value has been selected as the one to be used. The netBaltic system utilizes the link metric depending on an effective bandwidth offered by a given link, calculated over a considerable time interval to take into account propagation and traffic conditions, periodic link disruptions caused by vessel motion caused by waves etc. For the path metric to be an additive one, the link metric is inversely proportional to the effective bandwidth.

During normal operation of netBaltic node the value of the metric is calculated based on real-time measurements. In case of the presented numerical assessment it has been calculated as a function of distance, path loss and interface characteristics. Reference values for wireless link-rates considered in this assessment are depicted in Fig. 5.

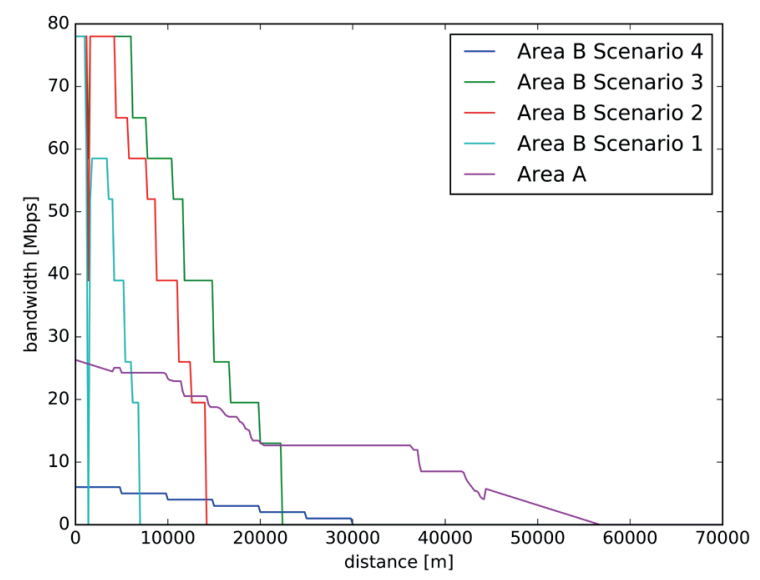

Fig. 5. The average effective bandwidth as a function of distance for evaluated wireless link types 
Cumulative metric for path is calculated as a sum of a sequence of links forming a route between the specific source node and the on-shore base station. The base station serving as a gateway for the node is chosen from all reachable base stations by selecting the one which offers the maximum upper bound on the effective bandwidth over the path.

Results obtained for system topologies based on real-world maritime vessel locations are presented in Figs. 6-11. The base stations are marked with yellow $\mathrm{X}$, blue dots represent nodes left in zone $\mathrm{C}$ due to their inability to communicate connected with any base station in real-time, while colored dots (in range from white to red) represent vessels connected to the Internet by mechanisms of zone A or B. Their specific color refers to the estimated maximum ship-to-shore transmission throughput.

Evaluation results show that the obtained network topology is relatively static and the number of vessels in each zone does not vary significantly in time, regardless of the analyzed scenario (see Fig. 6). Duration of the evaluation time equal to one day was chosen as a value interesting from the perspective of e-navigation services providers and delay-tolerant network mechanisms. In the presented analysis, vessels mobility is simulated by means of real data from AIS system. Data used in the evaluation is delivered by the Maritime Office. Thus, presented results refer to realistic vessel locations and their mobility model. However, as expected, the number of nodes in each zone strongly depends on the employed communication technology (and more specifically on its communication range).

For Scenario 1, where participating vessels are equipped only with the most rudimentary antennas, zone A contains almost all of nodes capable of communication with the shore (about 1200 nodes) - only about 200 nodes (i.e., about 5\% of all analyzed nodes) are provided with such connectivity outside of zone A (by multi-hop mesh network mechanisms of zone B, Fig. 6). However, it should be noted, that areas where zone $\mathrm{B}$ connectivity became available cover places with the highest concentration of vessels, such as bays, inlets and harbor approaches. They are also the places, where the demand for communication and its utility (for example for e-navigation, logistics or administrative purposes) can be expected to be high.

When analyzing other scenarios, it can be observed that gains in a communication range of participating vessels result in fast growth of the number of nodes capable of ship-toshore communication due to their participation in zone $\mathrm{B}$ of the system.

Scenarios 2 and 3 represent a case, where the off-the shelf RADWIN device has been additionally equipped with:

- in case of Scenario 2 - a set of directional antennas being an equivalent to a good quality, relatively high-gain omnidirectional antenna being able to fulfill its functions in maritime conditions (including, for example, some degree of resistance to wave-induced vessel movement),

- in case of Scenario 3 - an antenna installation described in Scenario 2, with a high power transmitter and a high sensitivity receiver.
The above scenarios utilize omnidirectional antennas available on the market, which achieve high gain as a result of their internal design. In the case of Scenario 4, the number of internal antenna elements is as great as sixty. However, netBaltic mechanisms offer the ability to transparently integrate many available communication devices, which allow many independent radio transceivers equipped with directional antennas to be employed to obtain omnidirectional communication capability within a single technology. Scenarios 2 and 3 can be considered as a case, where a set of off-the-shelf communication devices has been deployed in such a manner.

It can be observed, that in case of Scenario 2 and Scenario 3 the number of vessels connected directly to on-shore base stations is lower compared to Scenario 1. This effect is caused by a higher bandwidth being available to the node by using a two-hop path through zone B (utilizing high throughput WMAN zone B links), than a one-hop, direct zone A link at a particular range. Such an effect is not observed in Scenario 4, where in place of a relatively short range/high-bandwidth WMAN TDMA, a longrange communication technology (Kongsberg Maritime Broadband Radio) is used for links between vessels within zone B. In this case, their offered effective bandwidth is smaller and, in general, one-hop communication with onshore infrastructure is preferable.

Scenario 2 (Fig. 8) shows $18 \%$ of the total number of considered vessels being capable of bidirectional ship-toshore communication, which is a considerable improvement compared to $5 \%$ in case of simple deployment of the off-theshelf hardware assessed in Scenario 1. The result indicates, that introduction of a relatively simple improvement in node deployment can provide significant gains. Even more interesting are results of Scenario 3 (Fig. 9) where zone B comprises of $43.7 \%$ of all considered vessels, leaving only $26.3 \%$ of them without real-time, bidirectional, ship-toshore communication. This assessment illustrates, that the currently available off-the-shelf hardware is, most-likely, capable of being successfully employed in netBaltic system to significantly extend the area of broadband data transmission network coverage over the Baltic Sea.

The, already mentioned, Scenario 4 (Fig. 10) which employs a relatively low-throughput, long-range communication technology provides the most through coverage, with more than $80 \%$ of the netBaltic system nodes are able to obtain connectivity by employing networking mechanisms of zones A and B. However, it should be noted, that the connectivity provided in this case, is not a high throughput one and that the gain in the number of communication capable vessels as compared to Scenario 3 is less than $10 \%$.

With such a high importance of parameters provided by transmission technologies employed, it is important to note, that the netBaltic system is capable of transparently utilizing new solutions of this type, as long as they are capable of IP protocol support. In this situation, it can be expected that the system will naturally evolve to provide even better coverage 
with a steady development of new transmission technologies, observable today.

We should also remember, that nodes which are not capable of ship-to-shore connectivity, can often form separated groups of mesh-connected system nodes, thus obtaining a relatively long range communication capability with other such vessels. Normalized histograms presenting the average numbers of neighbors for examined scenarios are depicted in Fig. 11. As it can be seen, with increasing range of available wireless communication technologies, the share of nodes with greater number of neighbors is also increasing (visible especially for number of neighbors of about 110). In practice, wireless systems offering longer communication distance are not only more suitable for networking mechanism creating mesh networks, but also offer the ability to form clusters with direct communication between vessels. Moreover, as described in netBaltic System Architecture Section, dedicated Delay Tolerant Network mechanisms and applications have been designed by the netBaltic project to address such cases, which also can be considered as an important contribution of the project, further underlying its comprehensive approach in comparison with other maritime communication solutions.

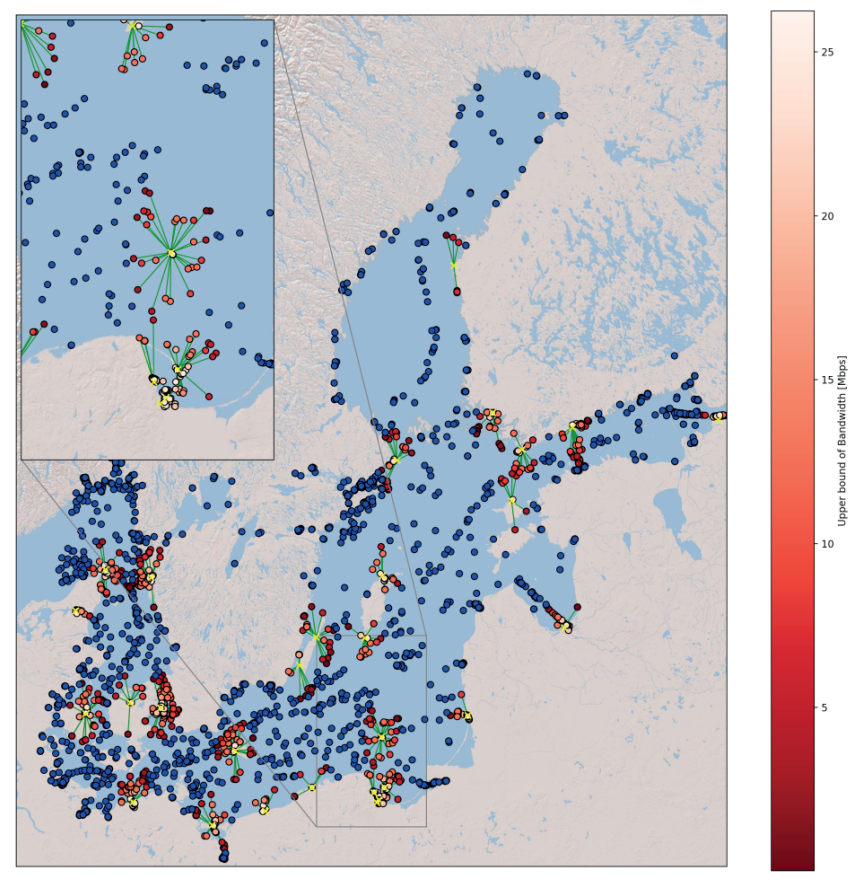

Fig. 7. Snapshot of netBaltic system topology - upper bound on bandwidth for Scenario 1
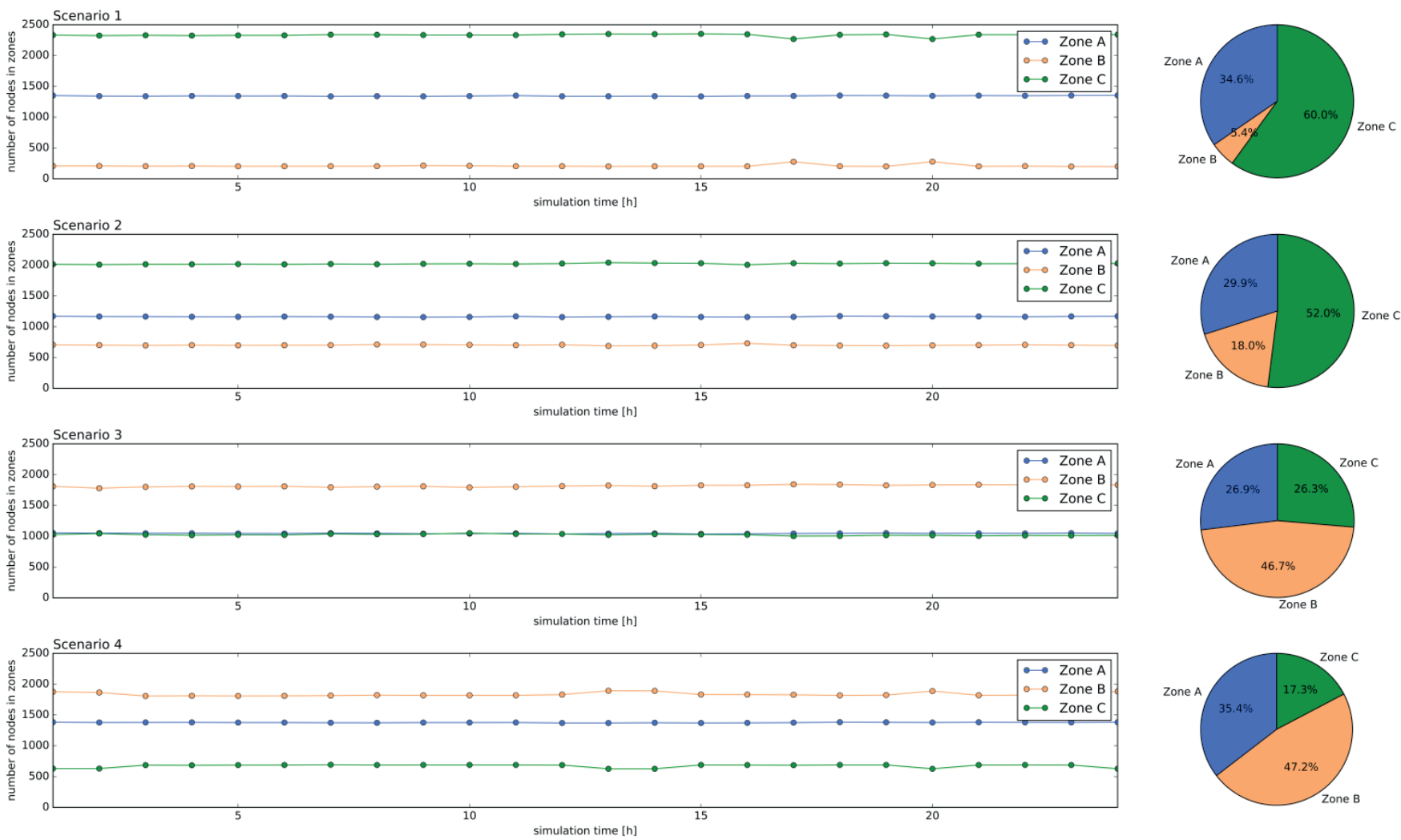

Fig. 6. Number of nodes in each zone of the netBaltic system as a function of simulation time (left part); size of netBaltic zones as a percentage of the total number of nodes (right part) 


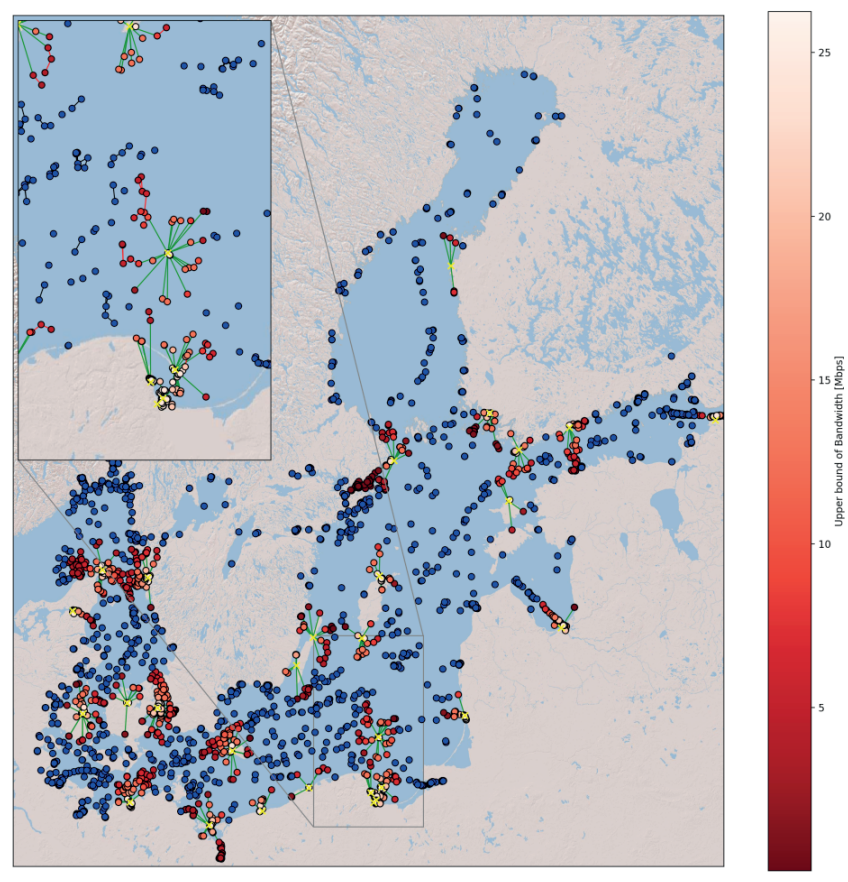

Fig. 8. Snapshot of netBaltic system topology - upper bound on bandwidth for Scenario 2

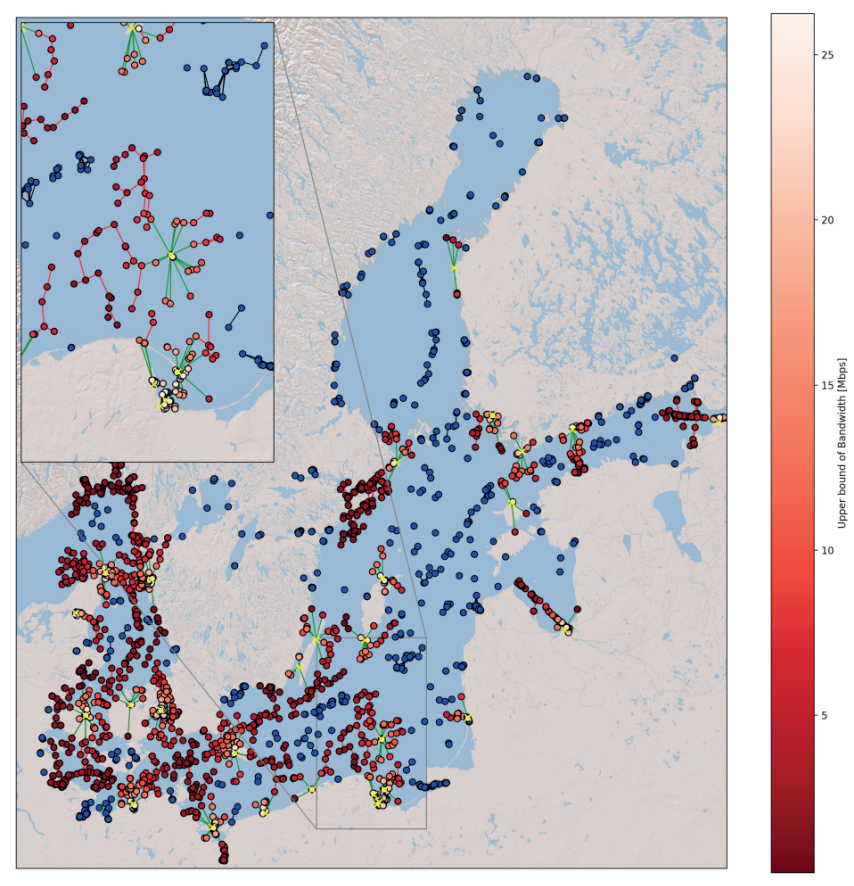

Fig. 9. Snapshot of netBaltic system topology - upper bound on bandwidth for Scenario 3

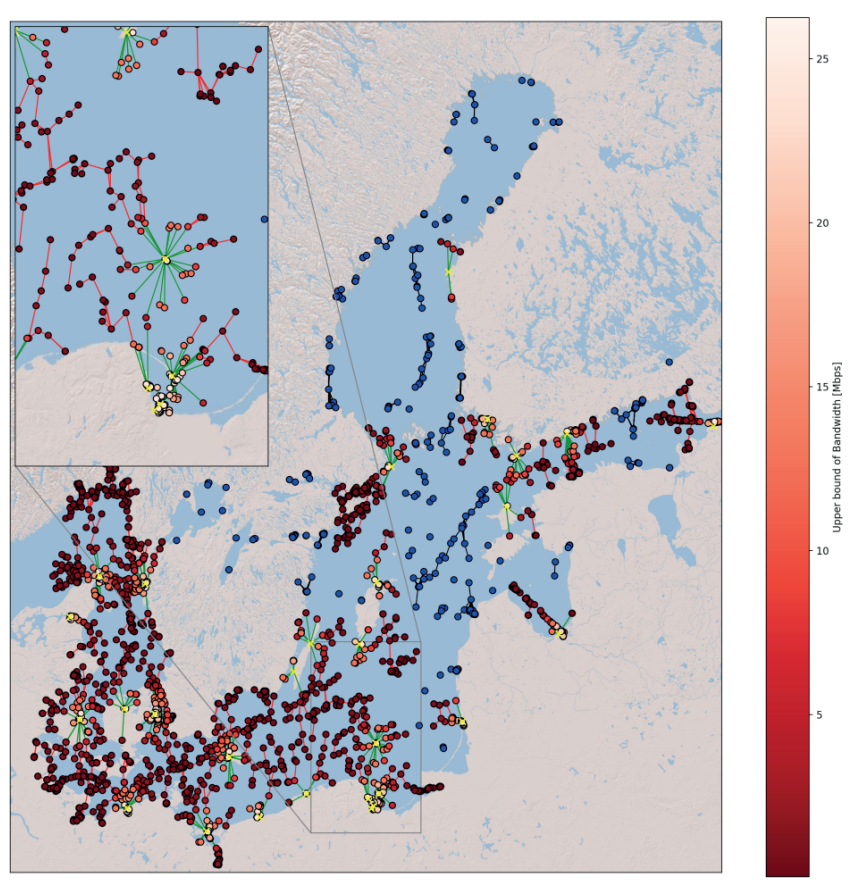

Fig. 10. Snapshot of netBaltic system topology - upper bound on bandwidth for Scenario 4
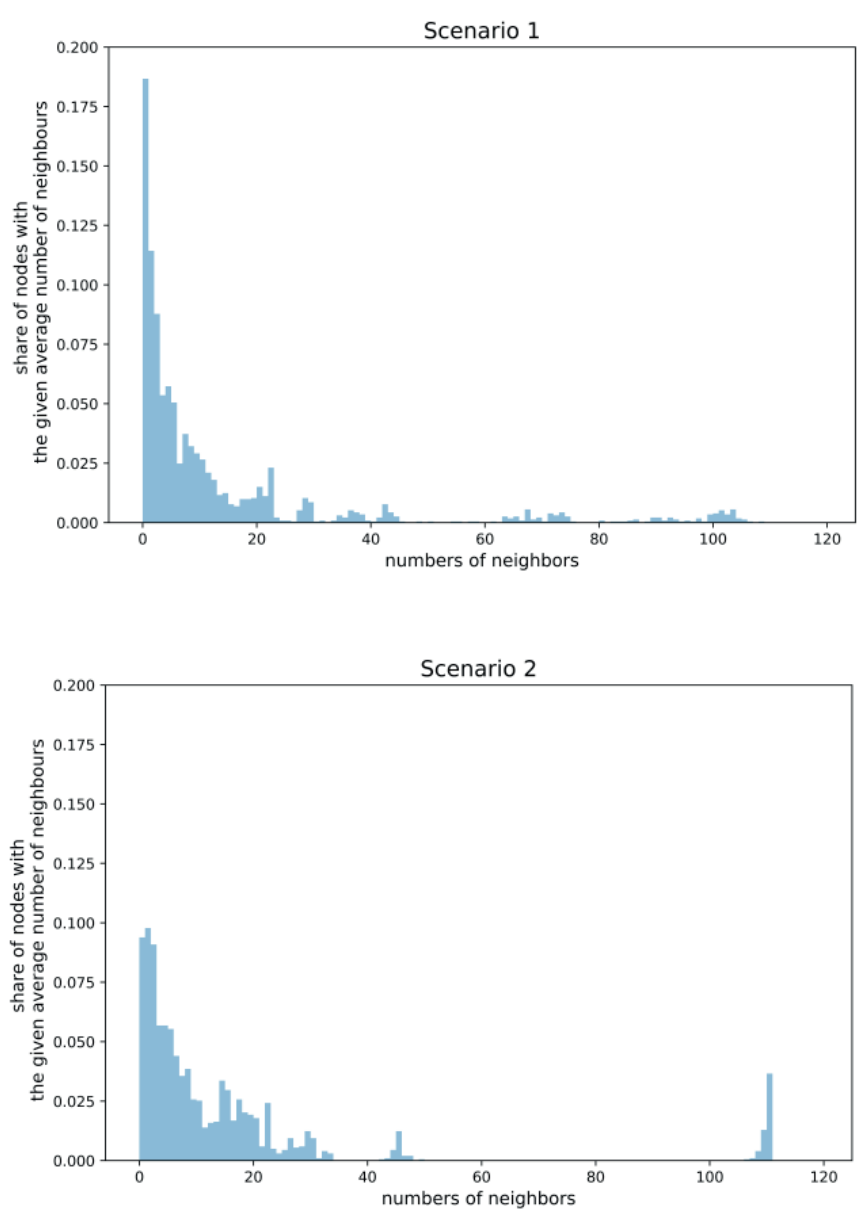

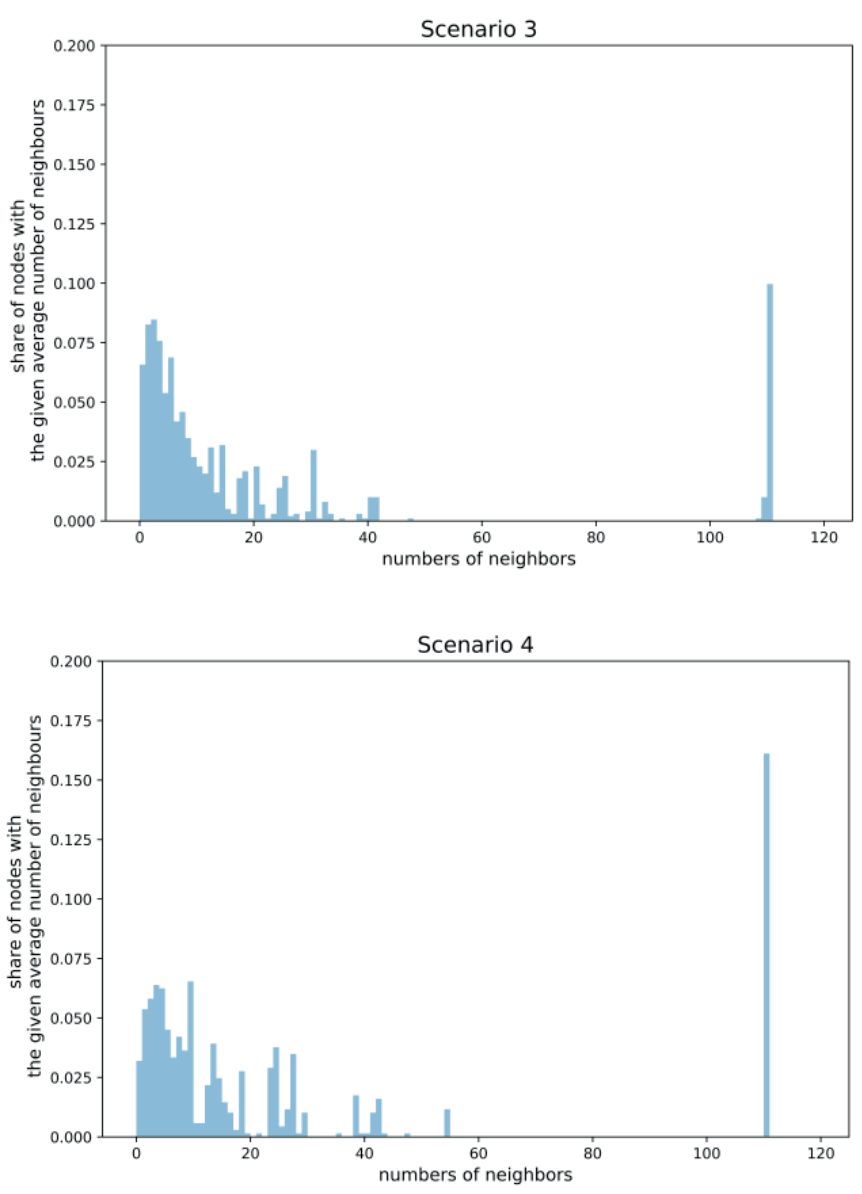

Fig. 11. Normalized histograms presenting numbers of neighbors in zone C for evaluated scenarios

\section{CONCLUSIONS}

In this paper, a new concept of a multi-zone, heterogeneous wireless maritime communication system was presented. Details of the proposed architecture of the netBaltic system were described highlighting the solutions introduced in the project that correspond to the specific maritime communication requirements. Numerical results of communication area coverages were discussed for four different scenarios of configuration of wireless interfaces of vessels. The results indicate that by employing the multi-zone, heterogeneous approach combined with appropriate wireless communication technologies the number of vessels being able to connect to the Internet can be significantly increased in comparison with traditional wireless systems (i.e., involving LTE one-hop communications only). The increase ranges from $14 \%$ for use of short-range communications technologies up to as high as $127 \%$, where long-range communication technologies are used in zone B. In the comparison, the number of nodes being able to communicate with onshore networks by means of mechanisms design for areas A and B is compared to the number of vessels being able to use only direct vessel - base station connections (area A).
It is also worth to notice, that the described heterogeneous approach allows the system to evolve by transparently utilizing new transmission technologies as they become available, which the presented results indicate as one of key elements influencing the ubiquity and quality of offered network access.

\section{ACKNOWLEDGMENT}

This work has been partially supported by the Applied Research Program under the Grant: ID PBS3/A3/20/2015 founded by the National Center for Research and Development. The infrastructure was supported by "PL-LAB2020" project, contract POIG.02.03.01-00-104/13-00 and "Future Internet Engineering” project, contract POIG.01.01.02-00-045/09-00.

\section{REFERENCES}

1. IMO MSC 81/23/10. "Work Programme. Development of an e-Navigation strategy", Submitted by Japan, Marshall Islands, the Netherlands, Norway, Singapore, the United Kingdom and the United States, International Maritime Organization, (2005)

2. J. Rak: „Resilient routing in communication networks”, Springer (2015)

3. K. S. Zaidi, V. Jeoti, A. Awang: „Wireless backhaul for broadband communication over sea" in Proc. IEEE 11th Malaysia International Conference on Comm., pp. 298-303 (2013)

4. „Working Document toward a draft new Report Maritime Radiocommunication Systems and Requirements". ITU RSG: IALA, e-NAV14-10.3.6 (2013)

5. A. Nowicki, M. Janecki, L. Dzierzbicka-Głowacka, M. Darecki, P. Piotrowski: The use of satellite data in the operational 3d coupled ecosystem model of the Baltic Sea (3d cembs), POLISH MARITIME RESEARCH 1(89) vol. 23 , pp. 20-24, (2016)

6. IMO NCSR Annex 7.: „Draft e-navigation strategy implementation plan" (2014) http://www.imo.org/en/ OurWork/Safety/Navigation/Documents/enavigation/SIP

7. L. Lambrinos, C. Djouvas: „Creating a maritime wireless mesh infrastructure for real-time applications", in Proc. IEEE GLOBECOM Workshops, pp. 529-532 (2011)

8. J. Arroyo: „Automatic Identification System”, (2011) http://www.nmea.org/Assets/nmea\%20collision\%20 avoidance\%20through\%20ais.pdf

9. GMDSS Manual, 2015 Edition - International Maritime Organization, (2015) 
10. ITU, „Maritime broadband wireless mesh networks”, report ITU-R M.2202, (2010)

11. J. Wozniak, K. Gierlowski and M. Hoeft, „Broadband communication solutions for maritime ITSs: Wider and faster deployment of new e-navigation services," 2017 15th ITST, Warsaw, pp. 1-11, (2017)

12. VDES: VHF Data Exchange System Overview. Workshop VDES Tokyo, (2014)

13. „Technical characteristics for a VHF data exchange system in the VHF maritime mobile band. M Series: Mobile, radiodetermination, amateur and related satellite services", Recommendation ITU-R M.2092-0, (2015)

14. „Development of VHF Data Exchange System (VDES)”, Report on IALA Workshop. Tokyo, Japan, (2016)

15. J. S. Pathmasuntharam et al.: „TRITON: High speed maritime mesh networks, „2008 IEEE 19th International Symposium on Personal, Indoor and Mobile Radio Communications, Cannes, pp. 1-5, (2008)

16. J. Wozniak, K. Gierlowski and M. Hoeft, „Broadband communication solutions for maritime ITSs: Wider and faster deployment of new e-navigation services," 2017 15th International Conference on ITS Telecommunications (ITST), Warsaw, 2017, pp. 1-11.

17. D.-S. Yoo, H.-J. Kim, J.-K. Choi, B.-T. Jang, S.-H. Ro: „A novel antenna tracking technique for maritime broadband communication (MariComm) system”, in Proc. ICACT2015 (17th International Conference on Advanced Communication Technology), pp. 225-229 (2015)

18. „IEEE Standard for Local and Metropolitan Area Networks Part 16: Air Interface for Fixed Broadband Wireless Access Systems," in IEEE Std 802.16-2004 (Revision of IEEE Std 802.16-2001), (2004)

19. „IEEE Standard for Local and metropolitan area networks Part 16: Air Interface for Broadband Wireless Access Systems Amendment 3: Advanced Air Interface," in IEEE Std 802.16m-2011 (Amendment to IEEE Std 802.16-2009) , vol., no., pp.1-1112, (2011)

20. M.-T. Zhou, V. D. Hoang, H. Harada: „TRITON: highspeed maritime wireless mesh network. IEEE Wireless Communications", vol. 20, no. 5, pp. 134-142 (2013)

21. R. Boreli, Y. Ge, T. Iyer, C. Dwertmann, J. S. Pathmasuntharam: „Intelligent Middleware for High Speed Maritime Mesh Networks with Satellite Communications", 9th int'l Conf. on ITS Telecomm., France (2009)
22. IEEE, „IEEE Standard for Information technology-Telecommunications and information exchange between systems Local and metropolitan area networks--Specific requirements - Part 11: Wireless LAN Medium Access Control (MAC) and Physical Layer (PHY) Specifications", IEEE Std 802.11-2016, 2016

23. „IEEE Standard for Information technology Telecommunications and information exchange between systems - Local and metropolitan area networks - Specific requirements - Part 11: Wireless LAN Medium Access Control (MAC) and Physical Layer (PHY) Specifications Amendment 4: Enhancements for Very High Throughput for Operation in Bands below $6 \mathrm{GHz}$ ", IEEE Std 802.11ac2013, (2013)

24. „IEEE Standard for Local and metropolitan area networks Part 16: Air Interface for Broadband Wireless Access Systems," in IEEE Std 802.16-2009 (Revision of IEEE Std 802.16-2004) pp.1-2080, (2009)

25. „IEEE Standard for Local and Metropolitan Area Networks Part 16: Air Interface for Fixed and Mobile Broadband Wireless Access Systems Amendment 2: Physical and Medium Access Control Layers for Combined Fixed and Mobile Operation in Licensed Bands and Corrigendum 1,” in IEEE Std 802.16e-2005 and IEEE Std 802.16-2004/ Cor 1-2005 (Amendment and Corrigendum to IEEE Std 802.16-2004), (2006)

26. Ubiquity Networks, AirMax Technical Description, https:// dl.ubnt.com/AirMax_ppt.pdf, retrieved 2017

27. RADWIN, FiberinMotion Wireless Mobility, http://www. radwin.com/products/fiberinmotion, retrieved 2017

28. M. Hoeft, P. Kaminski, J. Wozniak: „Logical Interface for Soft Handover - An Effective Scheme of Handovers in Proxy Mobile IPv6", 2015 8th IFIP Wireless and Mobile Networking Conference (WMNC), Munich, pp. 72-79, (2015)

29. M. Hoeft, K. Gierlowski, J. Wozniak: „Heterogeneous wireless communications system over the Baltic sea", Telecom. Review + Telecom. News 8-9, pp. 1196-1200, (2016) (in Polish).

30. M. Hoeft, K. Gierlowski, K. Nowicki, et al.: „netBaltic: Enabling Non-Satellite Wireless Communications over the Baltic Sea", Global Newsletter May 2016, pp. 2-4. In: IEEE Communications Magazine, (2016)

31. Perkins, C.; Belding-Royer, E.; Das, S., RFC 3561: Ad hoc On-Demand Distance Vector (AODV) Routing. IETF, 2003.

32. S.Kent, K. Seo, RFC4301 Security Architecture for the Internet Protocol. DOI: 10.17487/RFC4301, (2005) 
33. A.Vahdat, D. Becker: „Epidemic routing for partially connected ad hoc networks", Technical Report CS-2000-06, Department of Computer Science, Duke University, (2000)

34. K. Bronk, A. Lipka, R. Niski, B. Wereszko, K. Wereszko: „Measurement verification of the cellular systems' ranges achievable in the maritime environment", Telecom. Review + Telecom. News vol. 6, pp. 463-466, (2016) (in Polish)

35. Y. H. Lee, F. Dong, and Y. S. Meng, „Near sea-surface mobile radiowave propagation at $5 \mathrm{GHz}$ : measurements and modeling," Radioengineering, vol. 23, no. 3, pp. 824-830, (2014)

36. Kongsberg MBR 179 https://www.km.kongsberg.com/ks/ web/nokbg0397.nsf/AllWeb/6D9A832306BAC3F8C1257F CA0046926B/\$file/Datasheet_MBR179.pdf

\section{CONTACT WITH THE AUTHORS}

\section{Michał Hoeft}

e-mail: michal.hoeft@pg.edu.pl

Gdansk University of Technology

Faculty of Electronics, Telecommunications and Informatics,

G. Narutowicza 11/12, 80-233 Gdansk

\section{Poland}

\section{Krzysztof Gierłowski}

e-mail:krzgierl@pg.edu.pl

Gdansk University of Technology

Faculty of Electronics, Telecommunications and Informatics,

G. Narutowicza 11/12, 80-233 Gdansk

\section{Poland}

\section{Jacek Rak}

e-mail:jrak@pg.edu.pl

Gdansk University of Technology

Faculty of Electronics, Telecommunications and Informatics,

G. Narutowicza 11/12, 80-233 Gdansk

\section{Poland}

\section{Jozef Wozniak}

e-mail: jozwozni@pg.edu.pl

Gdansk University of Technology

Faculty of Electronics, Telecommunications and Informatics,

G. Narutowicza 11/12, 80-233 Gdansk

Poland 